\title{
Impact of Anxiety and Depression on Physical Health Condition and Disability in an Elderly Korean Population
}

\author{
Hee-Ju Kang', Kyung-Yeol Bae', Sung-Wan Kim¹, Hee-Young Shin², \\ II-Seon Shin ${ }^{1}$, Jin-Sang Yoon ${ }^{1}$, and Jae-Min Kim ${ }^{1 凶}$ \\ 1'Department of Psychiatry, Chonnam National University Medical School, Gwangju, Republic of Korea \\ ${ }^{2}$ Department of Biomedical Science, Chonnam National University Medical School, Gwangju, Republic of Korea
}

Objective This study aimed to investigate the longitudinal effects of anxiety, depression, and their comorbidity on physical disorders and disability in an elderly Korean population.

Methods In total, 1,204 community-dwelling elders were evaluated at baseline, and of these 909 (75\%) were re-assessed two years later. Anxiety and depression were identified at baseline using questions from the community version of the Geriatric Mental State diagnostic schedule (GMS-B3). Participants were assessed for functional disability and for 11 physical disorders both at baseline and at follow-up.

Results Anxiety alone was associated with the incidence of heart disease, depression alone with the incidence of asthma, and comorbid anxiety and depression with incidence of eyesight problem, persistent cough, asthma, hypertension, heart disease, and gastrointestinal problems. Comorbid anxiety and depression were associated with an increase in the number of physical disorders and the degree of disability during the two-year follow-up, compared to anxiety or depression alone or the absence of anxiety or depression.

Conclusion Anxiety, depression, and particularly their comorbidity should be assessed in the elderly population considering their longitudinal effects on physical disorders and disability. Future study is required to determine whether interventions aimed at these disorders can mitigate their impacts.

Psychiatry Investig 2017;14(3):240-248

Key Words Anxiety, Depression, Comorbid anxiety and depression, Physical disorders, Disability.

\section{INTRODUCTION}

The importance of mental health issues among the elderly has been recognized due to the current rapid aging of the population. Depression and anxiety are two of the most common mental health problems in later life and frequently co-occur. ${ }^{1}$ These psychiatric disorders are linked to considerable physical, cognitive, and overall functional disability and even mortality, ${ }^{1,2}$ and poorer prognosis has been found when anxiety and depression co-occur. ${ }^{3}$

To date, the impact of anxiety, depression and comorbid anxiety and depression on physical disorders has been studied

Received: March 1, 2016 Revised: April 24, 2016

Accepted: May 25, 2016 Available online: January 10, 2017

$\triangle$ Correspondence: Jae-Min Kim, MD, PhD

Department of Psychiatry, Chonnam National University Medical School, 160 Baekseo-ro, Dong-gu, Gwangju 61469, Republic of Korea

Tel: +82-62-220-6143, Fax: +82-62-225-2351, E-mail: jmkim@chonnam.ac.kr

(a) This is an Open Access article distributed under the terms of the Creative Commons Attribution Non-Commercial License (http://creativecommons.org/licenses/bync/4.0) which permits unrestricted non-commercial use, distribution, and reproduction in any medium, provided the original work is properly cited. primarily in working-age adult populations, and while these conditions are associated with the increased occurrence of physical disorders, ${ }^{4-6}$ it is uncertain whether these results can be generalized to the elderly, who typically suffer from a greater number of physical problems. ${ }^{7}$ There are currently only a limited number of prospective studies examining this relationship in the elderly: a few studies have assessed the impact of later-life depression on limited physical disorders such as cardiovascular disease and stroke and resulted in increased incidence of stroke or coronary heart disease during 1 to 15 years follow up; $;^{8,9}$ and one study examined the impact of later-life anxiety on physical disorders such as cardiovascular disease, gastrointestinal disorders, and arthritis in which, late-life anxiety increased incidence of various physical disorders but the kind of increased incident physical disorder were differed according to type of anxiety disorders. ${ }^{10}$ While prior literatures have examined the independent impact of anxiety and depression on physical disorders, few studies thus far has examined the impact of comorbid anxiety and depression on physical disorders in the elderly. 
Evidence suggests that sub-threshold symptoms that are insufficient to diagnose depression and anxiety disorders are frequent in elderly, ${ }^{11}$ and that these are associated with a chronic course and higher likelihood both of developing the disorder and of increased disability. ${ }^{12}$ Considering the high prevalence of comorbid anxiety and depression and the clinical importance of sub-threshold psychiatric symptoms among the elderly, this study aimed to investigate the longitudinal association between anxiety, depression and comorbid anxiety and depression including subthreshold symptoms, and physical disorders using data from a longitudinal community study of an elderly Korean population.

With respect to disability, it is well established that anxiety, depression and comorbid anxiety and depression including subthreshold level is associated with higher disability in latelife. However, prior literature has examined the independent impact of depression on disability in longitudinal study of elderly ${ }^{13}$ and studies investigating the impact of anxiety alone and comorbid anxiety and depression on disability were conducted mainly cross-sectionally. ${ }^{12,14,15}$ Therefore, we further aimed to examine changes in disability over the two-year follow-up period based on the baseline presence of anxiety and depression and their comorbidity (anxiety and depression).

\section{METHODS}

\section{Study overview and participants}

This study was carried out as a part of a larger prospective community-based study of late-life psychiatric morbidity in Gwangju, South Korea, from 2001 to 2003, the details of which have been described in a previous publication. ${ }^{16}$ All community residents aged 65 and over within two geographic catchment areas (one urban, one rural) were systematically identified from national registration lists and invited to participate at baseline, and those who participated were approached for follow-up two years later [mean \pm standard deviation (SD) interval: $2.4 \pm 0.3$ years]. No particular inclusion/exclusion criteria were applied. Home-based interviews with participants and their family members was carried out by trained graduated level research assistants, supervised by the project psychiatrist. Among the 1,566 inhabitants in registration lists, 1,204 inhabitants agreed to participate. No specific compensation was offered for participation. All participants provided formal written informed consent at each assessment point. This study was approved by the Chonnam National University Hospital Institutional Review Board.

\section{Diagnosis of anxiety and depression}

Anxiety and depression at baseline were assessed using the community version of the Geriatric Mental State Schedule
(GMS-B3) together with a diagnostic algorithm, the Automated Geriatric Examination for Computer-Assisted Taxonomy (AGECAT) ${ }^{17}$ These are structured diagnostic instruments used extensively in international epidemiological research on elderly populations. Cases of anxiety and depression were identified by AGECAT scores of $\geq 3$, which are thought to correspond to clinical levels of anxiety and depression warranting professional intervention. AGECAT scores of 1 or 2 were considered indicative of sub-threshold anxiety and depression; although these scores do not warrant routine professional intervention in a clinical context, they were considered meaningful in this context due to their association with disability in the elderly. ${ }^{12,18}$ Comorbid anxiety and depression was defined elderly with subthreshold/clinical level of anxiety and sub-threshold/clinical level of depression. In details, following four groups were included in comorbid anxiety and depression; subthreshold anxiety and subthreshold depression, subthreshold anxiety and clinical level of depression, clinical level of anxiety and subthreshold depression, and clinical level of anxiety and clinical levels depression.

\section{Evaluation for physical disorders and disability}

Physical disorders and functional disability were assessed both at baseline and at follow-up. Physical disorders were identified using a structured questionnaire which asked participants about the presence of 11 health problems common among the elderly: arthritis or rheumatism; eyesight problems; hearing difficulty or deafness; persistent cough; breathlessness, difficulty breathing or asthma; high blood pressure; heart disease or angina; gastric or intestinal problems; fainting or blackouts; paralysis, weakness, or loss of power in one leg or arm; and skin disorders such as pressure sores, leg ulcers, or severe burns. This questionnaire was specifically developed for estimating general health in elderly populations. ${ }^{19}$ Incidence of physical disorders was defined absent physical disorder at baseline but present follow-up 2 years later. In addition to the presence of individual disorders, the total number of conditions was also calculated. Disability was assessed using the Korean version of the World Health Organization Disability Assessment Schedule II (WHODAS II). ${ }^{20}$ WHODAS was developed to measure the impact of health conditions on function and disability of a patient considering impairments (affecting the body), activity limitation (affecting actions or behavior), and participants restriction (affecting experience of life). ${ }^{21}$ Moreover, additional advantage of the WHODAS II that it evaluated disability at the individual rather than disorder-specific status can facilitate assessing the total impact of the comorbid condition, ${ }^{22}$ which is particularly suited for elderly population suffered from several chronic disease. WHODAS II has been used for disability in elderly and has exhibited rigorous validity, reliability and cross- 
cultural applicability ${ }^{20,23-25}$ in which WHODAS scores was associated with functional capacity such as physical health, depression and cognitive function as well as functional ability such as muscle power impairment and speech impairment.

\section{Sociodemographic and clinical covariates at baseline}

A range of sociodemographic and clinical characteristics potentially associated with anxiety and depression were also measured at baseline. ${ }^{26}$ These included age, gender, years of education, area of residence (rural or urban), accommodation status (owned or rented), past occupation (manual or non-manual), current occupational status (employed or unemployed), monthly income (>\$200 USD or $\leq \$ 200$ USD or less), marital status (married or without spouse), physical inactivity, and cognitive function. Information was obtained from the participants and corroborated by a caregiver where possible. Daily physical activity was ascertained taking into consideration both work and leisure activity, and sedentary lifestyle was defined as a binary variable (active or inactive) ${ }^{27}$ Cognitive function was evaluated using the Mini-Mental State Examination (MMSE) ${ }^{28}$ and divided into two groups according to MMSE score (MMSE $<24$, lower cognitive function; MMSE $\geq 24$, higher cognitive function).

\section{Statistical analysis}

The distributions of anxiety and depressive states at baseline were tabulated, and for each condition, participants were classified into one of three categories: no anxiety/depression; subthreshold anxiety/depression; or clinical levels of anxiety/depression. Three categories of disorders were utilized: anxiety only, depression only, and comorbid anxiety and depression. A three by three table was created depicting the number and percent of participants in each category, by disorder classification as well as degree of each disorder. For the purpose of association analyses with physical disorders and disability, the categories for sub-threshold and clinical levels were collapsed into a single category for each disorder, as there were insufficient cases of clinically significant disorders to analyze the categories separately.

Univariate analyses ( $\chi^{2}$ tests) were conducted between baseline anxiety/depression status and each of the baseline charac- teristics measured; variables that showed significant associations $(\mathrm{p}<0.05)$ were considered to be covariates. For the analysis of incidence of physical disorders, the sample was restricted to those without any physical disorder at baseline. The associations between baseline anxiety/depression status and individual incident physical disorders were examined using the same logistic regression model, after controlling for baseline covariates. For analysis of change in the number of physical disorders and in the WHODAS scores, the changes between baseline and follow-up scores were calculated $(\Delta$ physical disorders $=$ total number of physical disorders at follow up-total number of physical disorders at baseline; $\triangle \mathrm{WHODAS}=\mathrm{WHODAS}$ scores at follow up-WHODAS scores at baseline). The associations between baseline anxiety/depression status and changes in the number of physical disorders and disability were examined using the same logistic regression model after controlling for covariates. Analyses were performed using the SPSS software package for Windows (version 21.0; IBM Inc., NY, USA).

\section{RESULTS}

\section{Recruitment and status of anxiety and depression}

A total of 1,204 respondents completed the baseline evaluation, and of these, 909 (75.5\%) were re-assessed at follow-up. The reasons for not followed participants were as follows; 102 participants could not be contacted, 61 participants died, 51 participants refused to participate, 46 participants changed address, 19 participants were too unwell to participate and 10 participants offered insufficient data about anxiety. Follow-up participants $(n=909)$ did not differ significantly at baseline from those not followed up $(n=295)$ with respect to age (mean $\pm S D, 72.4 \pm 5.7$ and $73.0 \pm 6.9$ years, respectively; $\mathrm{p}=0.394$ ), gender ( $58 \%$ and $59 \%$ female, respectively; $\mathrm{p}=0.711$ ), or psychological status (anxiety: 15\% and 16.3\%; depression: 9.5\% and $12.2 \%$; comorbid anxiety and depression: $22.8 \%$ and $23.1 \%$ respectively; $\mathrm{p}=0.437$ ). The distribution of baseline anxiety and depression status is summarized in Table 1 . Of the 1,204 total participants, 623 (51.7\%) did not suffer from either anxiety or depression, 184 (15.3\%) were identified with anxiety, 122 (10.2\%) were identified with depression, and 275 (22.8\%)

Table 1. Distribution of anxiety and depression at baseline

\begin{tabular}{lcccc}
\hline \multirow{2}{*}{ Anxiety } & \multicolumn{4}{c}{ Depression } \\
\cline { 2 - 5 } & No depression & Sub-threshold depression & Depression caseness & Total \\
\hline No anxiety & $623(51.7)$ & $97(8.1)$ & $25(2.1)$ & $745(61.9)$ \\
Sub-threshold anxiety & $153(12.7)$ & $112(9.3)$ & $86(7.1)$ & $351(29.1)$ \\
Anxiety caseness & $31(2.6)$ & $36(3.0)$ & $41(3.4)$ & $108(9.0)$ \\
Total & $807(67.0)$ & $245(20.4)$ & $152(12.6)$ & $1,204(100)$ \\
\hline
\end{tabular}

Data are number (\%) 
suffered from comorbid anxiety and depression. Comorbidity among those identified with at least one disorder was high; of those with depression, $69.3 \%$ also had anxiety, and $59.9 \%$ of those with anxiety also suffered from depression.

\section{Covariates of anxiety and depression status}

Univariate analyses between anxiety/depression status and the various sociodemographic and clinical characteristics were conducted and summarized in Table 2. Variables associated with baseline anxiety and depression status were older age ( $>75$ years), female sex, no education, urban residence, rented housing, no current employment, lower income ( $<$ \$200 USD/month), unmarried status, physical inactivity, and lower cognitive function $(\mathrm{MMSE}<24)$.

\section{Associations with incident physical disorders}

The incidence of physical disorders was assessed during follow-up, and the distribution of incident physical disorders by anxiety and depression status at baseline is shown in rows 1 through 11 of Table 3. At follow-up, only 30 individuals (2.5\%) had no physical disorders, and the remaining 879 (97.5\%) had one or more physical disorders. Adjusted associations derived from logistic regression analyzing the relationships between individual incident physical disorders and anxiety/depression status at baseline are described in Table 4. Having anxiety alone at baseline was significantly associated with the occurrence of heart disease; having depression at baseline was significantly associated with the occurrence of asthma; and comorbid anxiety and depression at baseline were associated with increased occurrence of eyesight problem, persistent cough, asthma, hypertension, heart disease, and gastrointestinal problems.

\section{Changes in the number of physical disorders and disability}

Changes in the total number of physical disorders between the two study points (the total number of physical disorders at follow up-the total number of physical disorders at baseline) were also calculated, and yielded a range of -1 to +8 . At followup, the total number of physical disorders remained unchanged in 307 (25.5\%) of the 909 follow-up participants; 267 (22.2\%) individuals developed one additional disorder during the follow-up period, $166(13.8 \%)$ participants developed two new disorders, and 140 (11.6\%) developed three or more new disorders during the study period. In contrast, 29 individuals (2.4\%) saw their physical disorders reduced by one during this period. With respect to disability, WHODAS scores (mean \pm SD) were $6.6 \pm 10.79$ at baseline and $20.6 \pm 20.49$ at follow-up. The unadjusted association of anxiety/depression status with changes in the number of physical disorders and disability scores over the two-year period is shown in Figure 1. Increases in the mean number of physical disorders during the followup period occurred in the following order: no anxiety/depression < anxiety only < depression only <comorbid anxiety and depression $(\mathrm{F}=8.555 ; \mathrm{p}<0.001)$. A similar pattern was found with respect to changes in WHODAS scores $(\mathrm{F}=4.679$; $\mathrm{p}<0.003)$. When associations for psychological status with changes in the number of physical disorders and WHODAS were adjusted, only the increase for the mean number of physical disorders and mean WHODAS scores among those with comorbid anxiety and depression were found to be statistically significant when compared to increases in scores for those with no anxiety or depression (last two rows of Table 4).

Table 2. Baseline characteristics by anxiety and depression status $(N=1,204)$

\begin{tabular}{lcccrrr}
\hline \multicolumn{1}{c}{ Baseline variables } & $\begin{array}{c}\text { No anxiety no } \\
\text { depression }(\mathrm{N}=623)\end{array}$ & $\begin{array}{c}\text { Anxiety only } \\
(\mathrm{N}=184)\end{array}$ & $\begin{array}{c}\text { Depression only } \\
(\mathrm{N}=122)\end{array}$ & $\begin{array}{c}\text { Comorbid anxiety } \\
\text { and depression }(\mathrm{N}=275)\end{array}$ & $\begin{array}{c}\chi^{2} \\
\text { p-value }\end{array}$ \\
\hline Age, 75+ years & $172(27.6)$ & $67(36.4)$ & $45(36.9)$ & $120(43.6)$ & 23.70 & $<0.001$ \\
Gender, women & $311(49.9)$ & $112(60.9)$ & $75(61.5)$ & $201(73.1)$ & 43.65 & $<0.001$ \\
Education, absent & $226(42.7)$ & $91(49.5)$ & $63(51.6)$ & $154(56.0)$ & 14.83 & 0.002 \\
Living area, urban & $196(31.5)$ & $57(31.0)$ & $60(49.2)$ & $145(52.7)$ & 46.93 & $<0.001$ \\
Housing, rented & $29(4.7)$ & $17(9.2)$ & $12(9.8)$ & $50(18.2)$ & 42.91 & $<0.001$ \\
Past occupation, manual & $550(88.3)$ & $167(90.8)$ & $111(91.0)$ & $255(92.7)$ & 4.54 & 0.209 \\
Current employment, no & $290(46.5)$ & $103(56.0)$ & $78(63.9)$ & $191(69.5)$ & 45.20 & $<0.001$ \\
Monthly income, $<200$ USD & $377(60.5)$ & $123(66.8)$ & $78(63.9)$ & $193(70.2)$ & 8.50 & 0.037 \\
Marital status, not married & $219(35.2)$ & $83(45.1)$ & $57(46.7)$ & $147(53.5)$ & 28.64 & $<0.001$ \\
Physical activity, inactive & $101(16.2)$ & $56(30.4)$ & $49(40.2)$ & $146(53.1)$ & 133.9 & $<0.001$ \\
MMSE, $<24$ & $263(42.2)$ & $95(51.6)$ & $78(63.9)$ & $178(64.7)$ & 48.17 & $<0.001$ \\
\hline
\end{tabular}

Data are number (\%). MMSE: Mini-Mental State Examination 


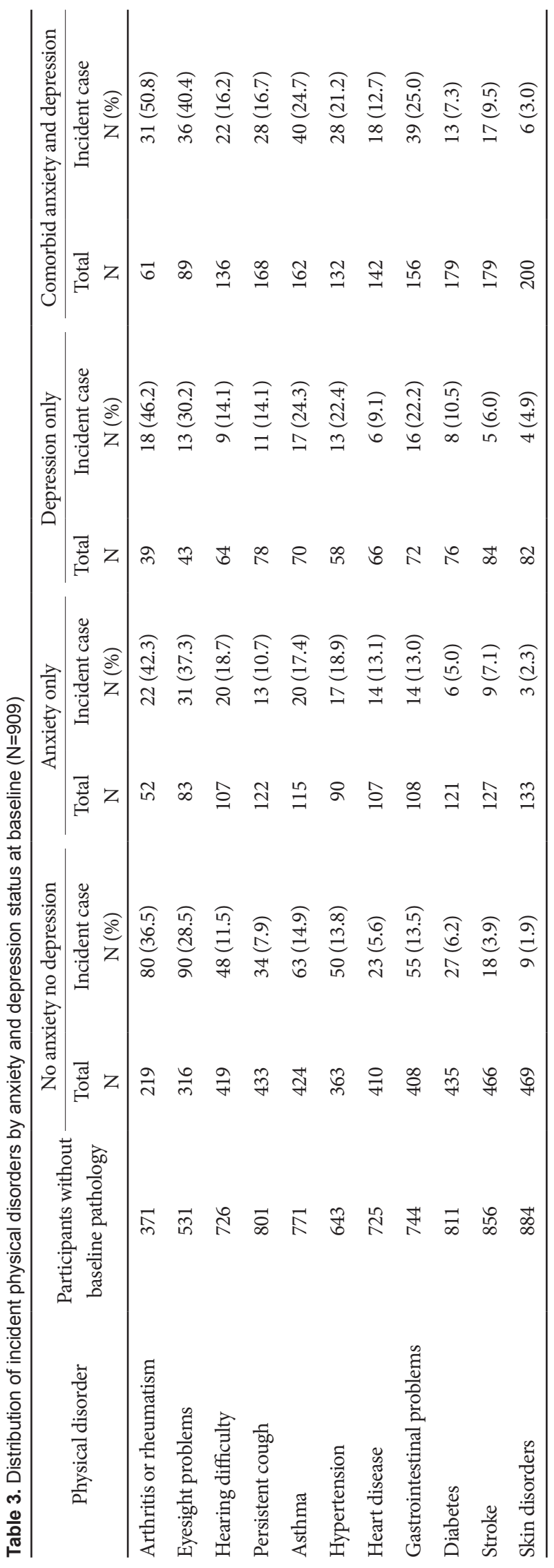

\section{DISCUSSION}

The principal findings of this 2-year longitudinal study of community elders were that: anxiety, depression and their comorbidity were highly prevalent in Korean elderly and associated with increased incidence of different physical disorders; and comorbid anxiety and depression is associated with more increase in number of physical disorders and with more disability after two years compared to anxiety and depression alone as well as no anxiety and depression after adjustment.

The prevalence of anxiety, depression, and their comorbidity in our sample were high $(31.2 \%, 33.0 \%$, and $22.8 \%$, respectively), consistent with previous studies of the elderly that found ranges of $15-52.3 \%$ for anxiety, ${ }^{29} 2.5-49 \%$ for depression, ${ }^{30}$ and $25-65 \%$ for comorbidity. ${ }^{31}$ Although the prevalence of later-life anxiety and depression varies according to study design, time frame, and diagnostic method, it is well-established that anxiety and depression are common among the elderly, and thus recognizing and managing these conditions appropriately is essential. ${ }^{1}$

The primary findings of our study were that comorbid anxiety and depression among the elderly were associated with an increase in the occurrence of various physical disorders including eyesight problem, persistent cough, hypertension, and gastrointestinal problems, in addition to heart disease and asthma which were also associated with anxiety alone and depression alone, respectively. Despite the of lack of previous prospective studies to evaluate the impact of comorbid anxiety and depression on the development of these physical disorders in an elderly population, longitudinal studies have reported significant correlations between psychological distress including anxiety or depression and the increased risk of these physical disorders among the general population, and occasionally the elderly population as well. Consistent with our findings, a recent meta-analysis found that depression increased the rate of hypertension in the general population. ${ }^{32}$ With respect to gastrointestinal disease, anxiety and depression in adults were suggested as significant independent predictors not only of functional gastrointestinal disorders, such as irritable bowel disease and functional dyspepsia, ${ }^{33}$ but also structural gastrointestinal disorders such as Crohn's disease and peptic ulcer. ${ }^{34}$ Additionally, a significant dose-response relationship, which means that adults with more co-occurring psychological disorders were associated with increased onset of new gastrointestinal symptoms, was reported in a general adult population. ${ }^{35} \mathrm{Re}-$ cently, the prospective association between anxiety disorders and the incidence of gastrointestinal disease was also suggested in an older population..$^{10}$ Moreover, depression was found to be associated with visual function loss in a 10-year follow-up study of community-dwelling persons aged 65 years and over. ${ }^{36}$ 
Because elderly with comorbid anxiety and depression are at increased risk for developing various physical disorders, intensive management, including psychological intervention and close monitoring for physical disorders, is warranted.

It was also found that elderly with comorbid anxiety and depression had the highest increase in number of physical disorders and highest increase in level of disability, followed by elderly people with depression or anxiety alone. Although previous cross-sectional studies have found that comorbid anxiety and depression are linked to greater functional disability and lower well-being than having depression or anxiety alone, ${ }^{12,14}$ this is the first study to investigate the longitudinal im- pact of comorbid anxiety and depression on changes in various physical disorders. There are several plausible mechanisms underlying this association. First, comorbid anxiety and depression among the elderly are associated with more severe psychopathology including greater suicidality, ${ }^{1,37}$ which may lead to poorer self-care and increased high-risk health behaviors such as smoking, physical inactivity, and non-adherence to medication or secondary prevention measures. These behaviors may in turn augment the risk of various physical disorders. Secondly, elderly with comorbid anxiety and depression report higher psychological distress, nearly 50\% more disability, and more somatic symptoms, including chest discomfort,

Table 4. Effects of anxiety and depression status at baseline on incident physical disorders ( $N=909)$

\begin{tabular}{lcccc}
\hline \multicolumn{1}{c}{ Physical disorder/status } & $\begin{array}{c}\text { Number of participants } \\
\text { without baseline pathology }\end{array}$ & Anxiety only & Depression only & $\begin{array}{c}\text { Comorbid anxiety } \\
\text { and depression }\end{array}$ \\
\hline Arthritis or rheumatism & 371 & $1.07(0.78-1.48)$ & $1.05(0.72-1.51)$ & $1.23(0.89-1.71)$ \\
Eyesight problems & 531 & $1.23(0.94-1.60)$ & $1.03(0.71-1.47)$ & $1.31(1.00-1.71)^{*}$ \\
Hearing difficulty & 726 & $1.28(0.96-1.72)$ & $1.10(0.74-1.63)$ & $1.15(0.85-1.56)$ \\
Persistent cough & 801 & $1.19(0.85-1.68)$ & $1.32(0.91-1.94)$ & $1.54(1.14-2.07)^{\dagger}$ \\
Asthma & 771 & $1.07(0.80-1.42)$ & $1.38(1.00-1.90)^{*}$ & $1.34(1.04-1.72)^{*}$ \\
Hypertension & 643 & $1.25(0.92-1.70)$ & $1.39(0.97-1.99)$ & $1.34(1.01-1.78)^{*}$ \\
Heart disease & 725 & $1.52(1.05-2.18)^{*}$ & $1.31(0.81-2.13)$ & $1.45(1.01-2.08)^{*}$ \\
Gastrointestinal problems & 744 & $0.94(0.68-1.29)$ & $1.35(0.98-1.87)$ & $1.43(1.10-1.85)^{\dagger}$ \\
Diabetes & 811 & $0.91(0.57-1.44)$ & $1.43(0.93-2.20)$ & $1.16(0.79-1.69)$ \\
Stroke & 856 & $1.35(0.88-2.06)$ & $1.14(0.67-1.93)$ & $1.44(0.98-2.10)$ \\
Skin disorders & 884 & $1.01(0.52-1.99)$ & $1.35(0.72-2.56)$ & $1.09(0.62-1.93)$ \\
Changes in the number of physical disorder & $909^{\mathrm{a}}$ & $1.10(0.95-1.28)$ & $1.27(1.07-1.51)^{\dagger}$ & $1.38(1.20-1.58)^{\ddagger}$ \\
Changes in WHODAS score & $909^{\mathrm{a}}$ & $1.00(0.99-1.01)$ & $1.01(1.00-1.35)$ & $1.02(1.00-1.02)^{*}$ \\
\hline
\end{tabular}

All ORs (95\% CIs) except changes in WHODAS scores were calculated after adjustment for age, gender, education, living area, accommodation status, current employment, monthly income, marital status, physical activity, and Mini-Mental State Examination score. With respect to changes in WHODAS scores, OR (95\% CIs) were calculated after adjustment for all covariates above and for total number of physical disorders. ${ }^{a}$ these two variables were analyzed in a total sample size of 909 , those who were followed two years later. ${ }^{*} \mathrm{p}<0.05$, ${ }^{\dagger} \mathrm{p}<0.01,{ }^{\ddagger} \mathrm{p}<0.001$. WHODAS: World Health Organization Disability Assessment Schedule, OR: odd ratio, CI: confidence interval
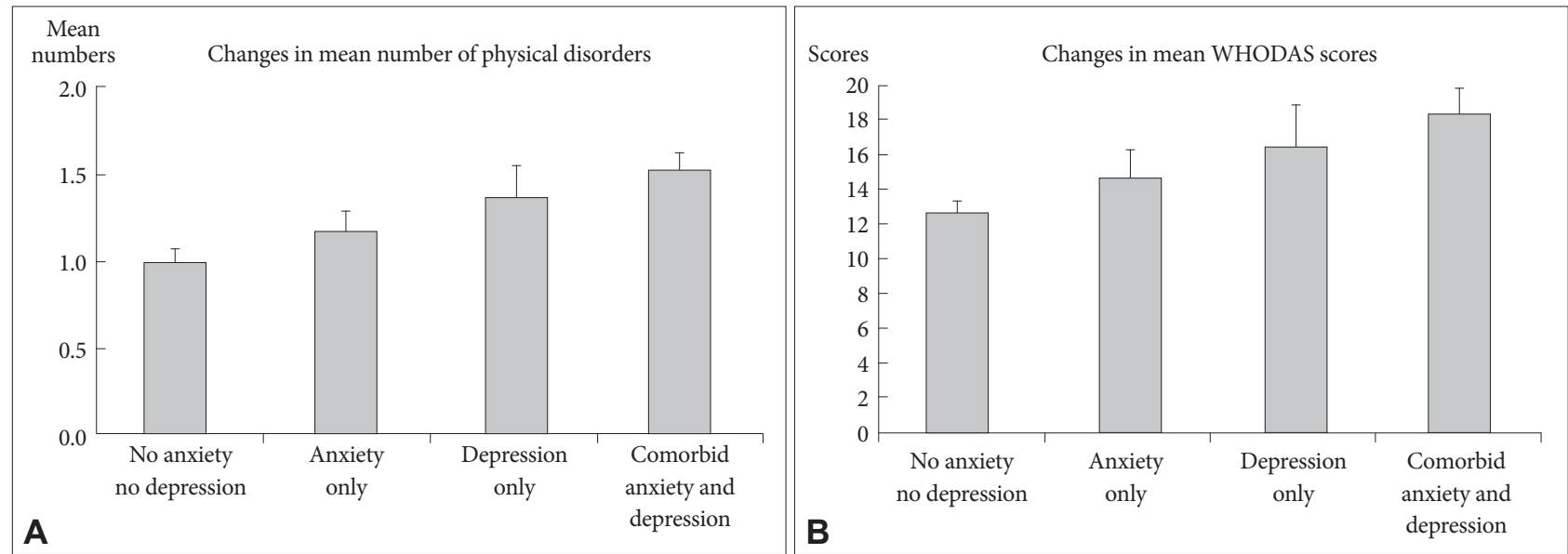

Figure 1. Flow diagram for effects of anxiety and depression status at baseline on changes in the number of physical disorders (A) and World Health Organization Disability Assessment Schedule (WHODAS) scores (B). 
palpitation, and gastrointestinal disturbance, than do those with individual disorders. ${ }^{14}$ Such prolonged physiological and psychological arousal from stress can impair body systems through hormonal or immunological mechanisms, ${ }^{38}$ increasing the risk of physical disease in elderly people who are physically fragile.

This study's finding that anxiety symptoms were associated with an increased incidence of heart disease at the two-year follow-up is consistent with previous studies of elderly populations that have found anxiety disorders, such as panic disorder and posttraumatic stress disorder, to be significantly associated with heart disease both cross-sectionally ${ }^{39}$ and longitudinally. ${ }^{10,40}$ The risk of developing heart disease among the elderly may be increased not only by the presence of specific anxiety disorders, but also by clinically relevant levels of anxiety symptoms, including those at sub-threshold levels which are associated with distress and adverse outcomes in elderly. ${ }^{41}$ Therefore, anxiety symptoms in the elderly and their longitudinal association with heart disease deserve greater attention.

The study also found that depressive symptoms were associated with greater risk of developing asthma. Although this is the first study to report a prospective association between depression and the incidence of asthma specifically in an elderly population, a meta-analysis of general adult participants followed for 8-20 years found that depression was associated with a $43 \%$ increased risk of developing asthma. ${ }^{42}$ Compared to general adult populations, the association between depression and the incidence of asthma among the elderly was evident even at sub-threshold levels of depressive symptoms, and this association was present despite the much shorter followup period.

Our study is the first to report a longitudinal association between psychological symptoms, including comorbid anxiety and depression, anxiety alone, and depression alone, and the incidence of various physical disorders in an elderly community cohort. We used standardized assessment scales including WHODAS II which has been used for measuring disability encompassing functional capacity and functional ability and has been exhibited rigorous validity, reliability and cross-cultural applicability. ${ }^{20,23-25}$ Moreover, our follow-up rate of $75.5 \%$ was adequate, and those who participated in the follow-up did not differ significantly from those who did not with respect to presence and number of physical disorders. Nonetheless, our study had several limitations. First, data on general physical health both at baseline and follow-up were based on participant self-reports, which may suffer from reporting bias. Although our finding of prevalence of individual physical disorders estimated by self-reports were high compared to previous finding estimated by disease registers, ${ }^{43}$ there is also prior evidence showing acceptable concordance between self-reports and medical. ${ }^{44}$ Additionally, medical records, including information regarding mental health treatment, were not available, and covariates such as personality traits were not included in the evaluation. Despite this, various other variables were considered, and several covariates of anxiety and depressive symptoms were identified. However, because anxiety and depression in the elderly are complex disorders, further studies investigating multidimensional covariates are required. ${ }^{2,45}$ Second, the two-year follow-up time frame was relatively short and may fail to capture physical disorders developing beyond this point. Moreover, our longitudinal analysis considered physical disorders to be an "incident" outcome based on 1 point follow up-i. e. absent on one occasion and present on another. Such an approach has only a limited ability to reflect physical disorder trajectories; for example, episode occurring and the disappearing between examination points would not be included in the analysis, which might result in underestimation of physical disorder incidence. Third, our measurement of anxiety was limited and did not identify specific anxiety subtypes, as the GMS diagnostic instrument is less well-validated with respect to the evaluation of anxiety than it is with regard to dementia or mood disorder assessment. ${ }^{17}$ Nevertheless, the GMS has proven to be useful in characterizing the psychopathology of anxiety, even at sub-threshold levels. ${ }^{12}$

In conclusion, anxiety, depression, and their comorbidity are common in elderly, and comorbidity between anxiety and depression is associated with greater risk of developing various physical disorders and higher levels of disability. Clinicians and community-based social service providers should seek to identify older patients with anxiety and depression those who are at risk for various physical disorders. Additionally, researchers and clinicians should keep in mind for managing elderly with collaborative care to improve general health condition in elderly population.

\section{Acknowledgments}

This study was supported by a grant of the Korean Health Technology R\&D Project, Ministry of Health \& Welfare, Republic of Korea (HI12C0003). The Ministry of Health and Welfare of Korea had paper publication design; in the collection, analysis and interpretation of data; in the writing of the report; or in the decision to submit the paper for publication.

\section{REFERENCES}

1. Lenze EJ, Mulsant BH, Shear MK, Alexopoulos GS, Frank E, Reynolds CF 3rd. Comorbidity of depression and anxiety disorders in later life. Depress Anxiety 2001;14:86-93.

2. Kang HJ, Kim SY, Bae KY, Kim SW, Shin IS, Yoon JS, et al. Comorbidity of depression with physical disorders: research and clinical implications. Chonnam Med J 2015;51:8-18.

3. Schoevers RA, Deeg DJ, van Tilburg W, Beekman AT. Depression and generalized anxiety disorder: co-occurrence and longitudinal patterns in elderly patients. Am J Geriatr Psychiatry 2005;13:31-39.

4. Bhattacharya R, Shen C, Sambamoorthi U. Excess risk of chronic 
physical conditions associated with depression and anxiety. BMC Psychiatry 2014;4:10.

5. Patten SB, Williams JV, Lavorato DH, Modgill G, Jetté N, Eliasziw M. Major depression as a risk factor for chronic disease incidence: longitudinal analyses in a general population cohort. Gen Hosp Psychiatry 2008;30:407-413.

6. Scott KM, Bruffaerts R, Tsang A, Ormel J, Alonso J, Angermeyer MC, et al. Depression-anxiety relationships with chronic physical conditions: results from the World Mental Health Surveys. J Affect Disord 2007;103:113-120.

7. Dall TM, Gallo PD, Chakrabarti R, West T, Semilla AP, Storm MV. An aging population and growing disease burden will require a large and specialized health care workforce by 2025. Health Aff (Milwood) 2013; 32:2013-2020.

8. Choi NG, Kim J, Marti CN, Chen GJ. Late-life depression and cardiovascular disease burden: examination of reciprocal relationship. Am J Geriatr Psychiatry 2014;22:1522-1529.

9. Brown JM, Stewart JC, Stump TE, Callahan CM. Risk of coronary heart disease events over 15 years among older adults with depressive symptoms. Am J Geriatr Psychiatry 2011;19:721-729.

10. El-Gabalawy R, Mackenzie CS, Pietrzak RH, Sareen J. A longitudinal examination of anxiety disorders and physical health conditions in a nationally representative sample of U.S. older adults. Exp Gerontol 2014; 60:46-56.

11. Gallo JJ, Lebowitz BD. The epidemiology of common late-life mental disorders in the community: themes for the new century. Psychiatr Serv 1999;50:1158-1166.

12. Prina AM, Ferri CP, Guerra M, Brayne C, Prince M. Co-occurrence of anxiety and depression amongst older adults in low-and middle-income countries: findings from the 10/66 study. Psychol Med 2011;41: 2047-2056.

13. Barry LC, Allore HG, Bruce ML, Gill TM. Longitudinal association between depressive symptoms and disability burden among older persons. J Gerontol A Biol Sci Med Sci 2009;64:1325-1332.

14. Cairney J, Corna LM, Veldhuizen S, Hermann N, Streiner DL. Comorbid depression and anxiety in later life: patterns of association, subjective well-being, and impairment. Am J Geriatr Psychiatry 2008;16:201-208.

15. Braam AW, Copeland JR, Delespaul PA, Beekman AT, Como A, Dewey $\mathrm{M}$, et al. Depression, subthreshold depression ad comorbid anxiety symptoms in older Europeans: results from the EURODEP concerted action. J Affect Disord 2014;155:266-272.

16. Kim JM, Stewart R, Kim SW, Kim SY, Bae KY, Kang HJ, et al. Physical health and incident late life depression: modification by cytokine genes. Neurobiol Aging 2013;34:356.

17. Copeland JR, Dewey ME, Griffiths-Jones HM. A computerized psychiatric diagnostic system and case nomenclature for elderly subjects: GMS and AGECAT. Psychol Med 1986;16:89-99.

18. Lyness JM, Kim J, Tang W, Tu X, Conwell Y, King DA, et al. The clinical significance of subsyndromal depression in older primary care patients. Am J Geriatr Psychiatry 2007;15:214-223.

19. Lindesay J. The Guy's/Age Concern Survey: physical health and psychiatric disorder in an urban elderly community. Int J Geriatr Psychiatry 1990;5:171-178.

20. Kim JM, Stewart R, Glozier N, Prince M, Kim SW, Yang SJ, et al. Physical health, depression and cognitive function as correlates of disability in an older Korean population. Int J Geriatr Psychiatry 2005;20:160-167.

21. World Health Organization. International Classification of Functioning, Disability, and Health (ICF). Geneva: World Health Organization; 2001.

22. Epping-Jordan JA, Ustun TB. The WHODAS-II: leveling the playing field for all disorders. WHO Mental Health Bull 2000;6:5-6.

23. von Korff M, Crane PK, Alonso J, Vilagut G, Angermeyer MC, Bruffaerts R, et al. Modified WHODAS-II provides valid measure of global disability but filter items increased skewness. J Clin Epidemiol 2008;61: 1132-1143.
24. Sousa RM, Dewey ME, Acosta D, Jotheeswaran AT, Castro-Costa E, Ferri CP, et al. Measuring disability across cultures--the psychometric properties of the WHODAS II in older people from seven low- and middle-income countries. The 10/66 Dementia Research Group population-based survey. Int J Methods Psychiatr Res 2010;19:1-17.

25. Chang KH, Liao HF, Yen CF, Hwang AW, Chi WC, Escorpizo R, et al. Association between muscle power impairment and WHODAS 2.0 in older adults with physical disability in Taiwan. Disabil Rehabil 2015;37: 712-720.

26. Vink D, Aartsen MJ, Schoevers RA. Risk factors for anxiety and depression in the elderly: a review. J Affect Disord 2008;106:29-44.

27. Prince M, Acosta D, Chiu H, Scasufca M, Varghese M; 10/66 Dementia Research Group. Dementia diagnosis in developing countries: a cross-cultural validation study. Lancet 2003;361:909-917.

28. Folstein MF, Fostein SE, McHugh PR. "Mini-Mental State". A practical method for grading the cognitive state of patients for the clinician. J Psychiatr Res 1975;12:189-198.

29. Bryant C, Jackson H, Ames D. The prevalence of anxiety in older adults: methodological issues and a review of the literature. J Affect Disord 2008;109:233-250.

30. Djernes JK. Prevalence and predictors of depression in populations of elderly: a review. Acta Psychiatr Scand 2006;113:372-387.

31. Kvaal K, McDougall FA, Brayne C, Matthews FE, Dewey ME; MRC CFAS. Co-occurrence of anxiety and depressive disorders in a community sample of older people: results from the MRC CFAS (Medical Research Council Cognitive Function and Ageing Study). Int J Geriatr Psychiatry 2008;23:229-237.

32. Meng L, Chen D, Yang Y, Zheng Y, Hui R. Depression increases the risk of hypertension incidence: a meta-analysis of prospective cohort studies. J Hypertens 2012;30:842-851.

33. Koloski NA, Jones M, Kalantar J, Weltman M, Zaquirre J, Talley NJ. The brain--gut pathway in functional gastrointestinal disorders is bidirectional: a 12-year prospective population-based study. Gut 2012; 61:1284-1290.

34. Ananthakrishnan AN, Khalili H, Pan A, Higuchi LM, de Silva P, Richter JM, et al. Association between depressive symptoms and incidence of Crohn's disease and ulcerative colitis: results from the Nurses' Health Study. Clin Gastroenterol Hepatol 2013;11:57-62.

35. Litcher-Kelly L, Lam Y, Broihier JA, Brand DL, Banker SV, Kotov R, et al. Longitudinal study of the impact of psychological distress symptoms on new-onset upper gastrointestinal symptoms in World Trade Center responders. Psychosom Med 2014;76:686-693.

36. Carrière I, Delcourt C, Daien V, Pérès K, Féart C, Berr C, et al. A prospective study of the bi-directional association between vision loss and depression in the elderly. J Affect Disord 2013;151:164-170.

37. Bartels SJ, Coakley E, Oxman TE, Constantino G, Oslin D, Chen H, et al. Suicidal and death ideation in older primary care patients with depression and anxiety and at risk alcohol use. Am J Geriatr Psychiatry 2002;10:417-427.

38. Schneiderman N, Ironson G, Siegel SD. Stress and health: psychological behavioral and biological determinants. Annu Rev Clin Psychol 2005; 1:607-628.

39. El-Gabalawy R, Mackenzie CS, Shooshtari S, Sareen J. Comorbid physical health conditions and anxiety disorders: a population-based exploration of prevalence and health outcomes among older adults. Gen Hosp Psychiatry 2011;33:556-564.

40. Kubzansky LD, Koenen KC, Spiro A 3rd, Vokonas PS, Sparrow D. Prospective study of posttraumatic stress disorder symptoms and coronary heart disease in the Normative Aging Study. Arch Gen Psychiatry 2007;64:109-116.

41. Mehta KM, Yaffe K, Brenes GA, Newman AS, Shorr RI, Simonsick EM, et al. Anxiety symptoms and decline in physical function over 5 years in the health, aging and body composition study. J Am Geriatr Soc 2007; 55:265-270.

42. Gao YH, Zhao HS, Zhang FR, Gao Y, Shen P, Chen RC, et al. The Re- 
lationship between depression and asthma: a meta-analysis of prospective studies. PLoS One 2015;10:e132424.

43. Barnett K, Mercer SW, Norbury M, Watt G, Wyke S, Guthrie B. Epidemiology of multimorbidity and implications for health care, research, and medical education: a cross-sectional study. Lancet 2012;380:37-43. 44. Kriegsman DM, Penninx BW, van Eijk JT, Boeke AJ, Deeq DJ. Self-reports and general practitioner information on the presence of chronic diseases in community dwelling elderly. A study on the accuracy of patients' self-reports and on determinants of inaccuracy. J Clin Epidemiol 1996;49:1407-1417.

45. Smith GS, Gunning-Dixon FM, Lotrich FE, Taylor WD, Evans JD. Translational research in late-life mood disorders: implications for future intervention and prevention research. Neuropsychopharmacology 2007;32:1857-1875. 